\title{
Aplicación seriada de paricalcitol intranodular ¿Es eficaz en el tratamiento del hiperparatiroidismo secundario refractario?
}

\author{
Serial intranodular application of paricalcitol \\ Is effective in the treatment of secondary refractory \\ hyperparathyroidism?
}

\author{
César Augusto Restrepo, Campo Elías Castillo, María Fernanda Sanz \\ - Manizales (Colombia)
}

\section{Resumen}

Objetivo: determinar la eficacia del paricalcitol en la reducción de los niveles de PTH intacta (PTHi) al aplicarlo directamente en las glándulas paratiroides en pacientes con enfermedad renal crónica estadio 5 (ERC E5) en terapia dialítica con hiperparatiroidismo refractario e hiperplasia nodular.

Material y métodos: pacientes con ERC E5 en terapia de diálisis e hiperparatiroidismo secundario refractario y en quienes por ecografía de cuello se detectó hiperplasia de más de una glándula paratiroides, caracterizada por un volumen mayor de $500 \mathrm{~mm}^{3}$. A cada paciente por guía ecográfica dirigida se le aplicó 0.5 cc (2.5 ugs) de paricalcitol (Zemplar®) en cada glándula hiperplásica independientemente de su tamaño, y según el grupo. En el grupo 1 (G1) recibieron paricalcitol semanal para un máximo de dos glándulas por sesión siempre del mismo lado y por un total de dos dosis por glándula; para el grupo 2 (G2) paricalcitol cada 15 días en esquema semejante al grupo 1 para un total de dos dosis por glándula y finalmente para el grupo 3 (G3) paricalcitol cada mes, pero en cada sesión se inyectaron todas las glándulas para un total de cuatro dosis por glándula.

Diseño: estudio de intervención sin grupo control.

Análisis estadístico: se evaluó si la intervención daba lugar a reducción significativa en los valores de PTHi, y las variables calcio, fósforo, calcio x fósforo y fosfatasa alcalina.

Resultados: en el G1 (seis pacientes) se encontró una disminución estadísticamente significativa entre el nivel promedio de PTHi inicial y PTHi a los 30 días de iniciado el tratamiento ( $\mathrm{p}=0.0077$ ), pero no a los 51 ni 81 días. En el G2 (seis pacientes) al comparar la PTHi inicial, con el valor detectado a los 51, 75, 105 y 135 días posaplicación de la última dosis de paricalcitol, no hubo cambios significativos. En el G3 (cuatro pacientes)con relación a la PTHi inicial hubo reducción significativa en sus valores a los 60 días $(\mathrm{p}=0.012), 120$ días $(\mathrm{p}=0.0099)$ y 180 días $(\mathrm{p}=0.00095)$, pero no a los 240 días $(\mathrm{p}=0.214049)$.

En las demás variables (calcio, fósforo, calcio x fósforo, fosfatasa alcalina) no se detectaron cambios significativos en ningún grupo. No se presentaron complicaciones importantes.

Conclusiones: en pacientes con ERC E5, en terapia de diálisis e hiperparatiroidismo secundario refractario, la administración de paricalcitol intranodular logra reducir significativamente los niveles de PTHi, siempre y cuando se administre en grupos de dos glándulas con un intervalo de tiempo no superior a una semana, o por administración mensual simultanea de todas las glándulas. (Acta Med Colomb 2015; 40: 125-131).

Palabras clave: enfermedad renal crónica, hiperparatiroidismo secundario, paricalcitol.

\section{Abstract}

Objective: go determine the effectiveness of paricalcitol in reducing the levels of intact PTH (iPTH) when applied directly on the parathyroid glands in patients with Grade 5 chronic kidney disease (CKD G5) with refractory hyperparathyroidism and nodular hyperplasia in dialysis therapy.
Dr. César Augusto Restrepo Valencia: Internista Nefrólogo. Profesor Asociado Universidad de Caldas, Profesor Catedrático Universidad de Manizales; Dr. Campo Elías Castillo Pinilla: Radiólogo, Profesor Universidad de Caldas; Sra. María Fernanda Sanz Scovino: Enfermera Jefe, Certificada en Nefrología. Manizales (Colombia).

Correspondencia. Dr. César Augusto Restrepo Valencia. Manizales (Colombia).

E-mail:caugustorv@une.net.co

Recibido: 23/VI/2014 Aceptado: 21/V/2015 
Materials and methods: CKD G5 patients on dialysis therapy with refractory secondary hyperparathyroidism in whom through neck echography hyperplasia of more than one parathyroid gland characterized by a volume greater than $500 \mathrm{~mm}^{3}$ was detected. Through directed ultrasound guidance to each patient $0.5 \mathrm{cc}$ (2.5ugs) of paricalcitol (Zemplar $囚$ ) was applied in each hyperplastic gland regardless of size and according to the group. Group 1 (G1) received weekly paricalcitol for up to two glands per session always on the same side, for a total of two doses per gland; Group 2 (G2) received paricalcitol every 15 days in similar scheme as group 1 for a total of two doses per gland and finally for group 3 (G3) paricalcitol each month, but in every session all the glands were injected for a total four doses per gland.

Design: intervention study with no control group.

Statistical analysis: an assessment whether the intervention resulted in significant reduction in iPTH and variables calcium, phosphorus, calcium x phosphorus and alkaline phosphatase, was made.

Results: In G1 (six patients), a statistically significant decrease between the average level of initial iPTH and iPTH at 30 days of starting treatment $(\mathrm{p}=0.0077)$, but not at 51 or 81 days was found. In G2 (six patients) by comparing the initial iPTH, with the detected value at 51, 75, 105 and 135 days post-application of the last dose of paricalcitol, there were no significant changes. In G3 (four patients) relative to the initial iPTH there was significant reduction in their values at 60 days $(\mathrm{p}=0.012), 120$ days $(\mathrm{p}=0.0099)$ and 180 days $(\mathrm{p}=0.00095)$, but not at 240 days $(\mathrm{p}=0.214049)$.

On the other variables (calcium, phosphorus, calcium x phosphorus, alkaline phosphatase), no significant changes were detected in either group. No major complications occurred.

Conclusions: in patients with CKD G5 in dialysis therapy and refractory secondary hyperparathyroidism, administration of intranodular paricalcitol achieves significantly lower levels of iPTH, as long as it is administered in groups of two glands with a time interval not exceeding one week, or by simultaneous monthly administration of all glands. (Acta Med Colomb 2015; 40:125-131).

Keywords: chronic kidney disease, secondary hyperparathyroidism, paricalcitol.

\section{Introducción}

El hiperparatiroidismo es una complicación común en pacientes con enfermedad renal crónica (ERC), y se asocia a gran variedad de complicaciones óseas y cardiovasculares (1). En su tratamiento en pacientes con ERC estadio 5 (ERC E5) en terapia de diálisis intervienen diversas variables incluidas entre otras niveles séricos de calcio, fósforo, producto calcio-fósforo, valores de PTH intacta (PTHi), anormalidades óseas y presencia de calcificaciones vasculares. Diversos medicamentos con diferentes mecanismos de acción participan en su terapia, pero a pesar de las terapias actuales se reporta que un número significativo de pacientes con hiperparatiroidismo secundario fallan en responder a la terapia con agonistas selectivos o no selectivos del receptor de la vitamina $\mathrm{D}$, y más recientemente se presentan reportes de falta de respuesta al cinacalcet (2). Lo anterior es consecuencia de la aparición de hiperplasia nodular de las glándulas paratiroides, lo cual les imprime un carácter autónomo con significativa reducción en el número de receptores a la vitamina $\mathrm{D}$ y calcio $(3,4)$. En este grupo de pacientes la paratiroidectomía constituye una alternativa terapéutica, pero en algunos pacientes por su comorbilidad cardiovascular es imposible practicarla. El etanol intranodular representa otra elección, pero recientemente confirmamos que su eficacia se limita a una sola glándula paratiroides hiperplásica (5).
Altos niveles locales intraglandulares de agonistas selectivos o no selectivos del receptor de la vitamina $\mathrm{D}$ podrían ocupar en forma sostenida el bajo número de receptores de vitamina D en glándulas con hiperplasia nodular y lograr de esta manera mayor eficacia que sus preparados orales e intravenosos. Hay informes controversiales sobre los resultados de este tipo de terapia, y consideramos que los mismos pueden ser consecuencia de no haber tenido en cuenta el número y tamaño de glándulas hiperplásicas que presenten los pacientes, ni persistir en su aplicación en el curso del tiempo. En este estudio nosotros decidimos investigar si la aplicación seriada intranodular de paricalcitol en pacientes con ERC e hiperplasia nodular de glándulas paratiroides podría controlar eficientemente la secreción glandular.

\section{Material y métodos}

Pacientes con ERC-E5 en diálisis (ERC-E5D) (hemodiálisis o diálisis peritoneal) mayores de 18 años, interconsultados al servicio de radiología por sospecha de hiperparatiroidismo secundario refractario, quienes presentaran valores de PTH intacta (PTHi) mayores a 600 $\mathrm{pg} / \mathrm{mL}$, a pesar de recibir terapia con dieta baja en fosfatos, quelantes de fosfatos orales y vitamina $\mathrm{D}$ activa (calcitriol) por un mínimo de 12 meses.

A todos los pacientes se les practicó ultrasonografía de cuello, tiroides-paratiroides con equipo de alta resolución 
(equipo Phillips Enviisor CHD - Transductor $12 \mathrm{mHz}$ ). Se incluyeron en el estudio pacientes en quienes se detectó hiperplasia de más de una glándula paratiroides, caracterizada por un volumen mayor de $500 \mathrm{~mm}^{3}$, calculado según la fórmula: a x b x c x $\pi / 6$ en $\mathrm{mm}^{3}$, donde a, b y c corresponden al diámetro de las tres dimensiones de la glándula, de acuerdo con las recomendaciones de Restrepo V y col (6). Fueron criterios de exclusión: discrasia de células sanguíneas y/o anormalidades en la coagulación, disfonía y/o parálisis de cuerdas vocales, alergia a agonistas de la vitamina $\mathrm{D}$, temor a punciones y no firmar aceptación del procedimiento.

Para los pacientes que aceptaron participar en el estudio, previa firma del consentimiento informado, se procedió en forma ambulatoria en sala de radiología a practicar asepsia de cuello, cubriendo esa área con campos estériles, revistiendo el traductor del ecógrafo con funda estéril, y luego bajo anestesia local y guía ecográfica dirigida se aplicaron 0.5 cc (2.5 ugs) de paricalcitol (Zemplarß) en cada glándula hiperplásica independientemente de su tamaño, y según el grupo. Los pacientes se dividieron en tres grupos simplemente por orden de llegada, y con el objetivo de lograr idealmente un número de seis por grupo: grupo 1 (G1) recibieron paricalcitol semanal para un máximo de dos glándulas por sesión siempre del mismo lado y por un total de dos dosis por glándula (tiempo total de intervención: 21 días); grupo 2 (G2) paricalcitol cada 15 días en esquema semejante al grupo 1 para un total de dos dosis por glándula (tiempo total de intervención 42 días) y finalmente grupo 3 (G3) paricalcitol cada mes, pero en cada sesión se inyectaron todas las glándulas para un total de cuatro dosis por glándula (tiempo de intervención 90 días). En el G1 los niveles de PTHi se evaluaron a la semana, 30 y 60 días posteriores a la última dosis de paricalcitol, en el G2 a la semana, 30 y 90 días posteriores a la última dosis y en G3 cada 60 días a partir de la primera dosis hasta por 240 días, valor final que correspondía también a 120 días posterior a la última dosis (se diferencia de los anteriores en que la PTH se evaluó durante el tratamiento y posterior al mismo). El calcio, fósforo y el producto calcio $\mathrm{x}$ fósforo se evaluaron cada mes, la fosfatasa alcalina en forma variable según el grupo. Se registraron los efectos colaterales asociados a cada uno de los procedimientos.

El estudio fue considerado de intervención sin grupo control.

La recolección de la información se llevó a cabo mediante una base de datos diseñada en Excel, la cual fue diligenciada por los investigadores, leída y procesada en el programa SPSS versión 15.0. Con los datos recopilados se obtuvieron indicadores descriptivos como el promedio aritmético y la desviación estándar. Para probar si hubo cambios significativos en las variables se hicieron pruebas de hipótesis para los promedios. En todos los casos se tomaron decisiones con un nivel de significancia de $5 \%$ como máximo. Cuando se plantearon hipótesis para diferencia de medias, teniendo en cuenta que las muestras con las que se trabajó son pequeñas y previa comprobación de la igualdad de varianzas, se utilizó la prueba t de Student. Cuando se hicieron análisis de varianza (ANOVA), se verificaron los supuestos de normalidad y de igualdad de varianzas. En aquellos casos en los cuales no se cumplió el supuesto de normalidad en los datos, se utilizó la prueba de Kruskall-Wallis que es la recomendada en estas situaciones.

Se consideró eficacia terapéutica completa lograr restaurar los niveles de PTH intacta a valores en rangos considerados satisfactorios $(150-300 \mathrm{pg} / \mathrm{mL})$ y parcial a una reducción de $50 \%$ de su valor inicial.

En ningún paciente se practicó gammagrafía con Sestamibi para identificar glándulas ectópicas.

El proyecto fue revisado y aprobado por el departamento clinicoquirúrgico y por el comité de Bioética de la Universidad de Caldas.

\section{Resultados}

Durante un periodo de dos años se evaluaron en la Universidad de Caldas y en el servicio de Radiología del Hospital Santa Sofía (Manizales-Caldas, Colombia-Sur América) 86 pacientes con ERC-E5-D (hemodiálisis o diálisis peritoneal), interconsultados por sospecha de hiperparatiroidismo secundario refractario, y con las características previamente anotadas. En 30 pacientes (34.88\%) se detectó hiperplasia de glándulas paratiroides, con volumen de las glándulas mayor de $500 \mathrm{~mm}^{3}$. Dieciséis pacientes aceptaron participar en el estudio, seis en el grupo 1 (G1), seis para el grupo $2(\mathrm{G} 2)$ y sólo se lograron incluir cuatro en el grupo 3 (G3).

El número promedio de nódulos infiltrados por grupos y el volumen glandular promedio (VGM) en $\mathrm{mm}^{3}$ fue: $\mathrm{G} 1$ : 3 nódulos, VGM: $684 \pm 137$; G2: 3.17 nódulos, VGM 664 \pm 148; G3: 2.,25 nódulos, VGM: $826 \pm 213$ (Figuras 1 y 2).

En el análisis estadístico del grupo 1 se encontró que había una disminución estadísticamente significativa entre el nivel promedio de PTHi inicial y PTHi a los 30 días de iniciado el tratamiento (Estadística de trabajo $($ EdeT $)=2.9141$, valor de $\mathrm{p}=0.00772783$ ). Al hacer la comparación entre el nivel promedio de PTHi inicial y PTHi a los 51 días (EdeT $=1,12256$, valor de $\mathrm{p}=0.2877$ ) y PTH a los 81 días (EdeT $=0.606979$, valor de $\mathrm{p}=0.557394)$ no se detectaron cambios significativos. Con el análisis de varianza (ANOVA), en las demás variables (calcio, fósforo, calcio x fósforo, fosfatasa alcalina) no se detectaron cambios significativos en el nivel promedio a través del tiempo (Tabla 1).

En el grupo 2 se encontró que para la PTHi, calcio, fósforo, calcio $\mathrm{x}$ fósforo y fosfatasa alcalina de su valor inicial y el detectado a los 51, 75, 105 y 135 días posaplicación de la última dosis de paricalcitol, no hubo cambios significativos en el nivel promedio de las variables a través del tiempo (Tabla 2).

En el grupo 3 se concluyó que había cambios significativos en al menos un promedio (EdeT=6.40, valor de $\mathrm{p}=0.0033$ ) de la PTHi a través del tiempo. Para determinar cuáles fueron los promedios diferentes, se plantearon prue- 


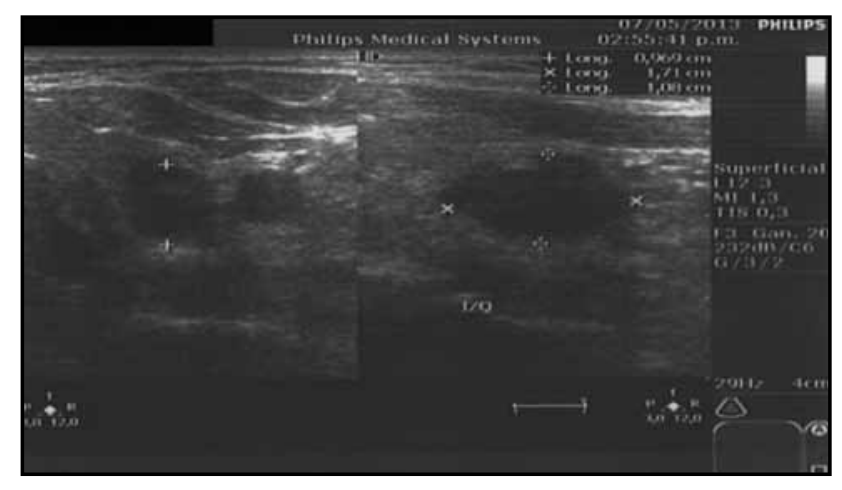

Figura 1. Adenoma paratiroideo por $937 \mathrm{~mm}^{3}$.

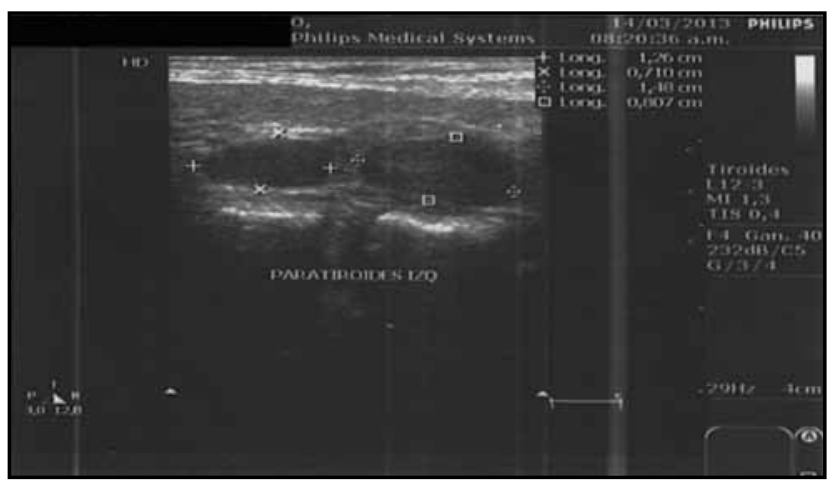

Figura 2. Dos adenomas paratiroideos de 351 y $586 \mathrm{~mm}^{3}$.

Tabla 1. Grupo 1. Promedio y desviacion estandar del grupo 1 (Tamaño de muestra seis pacientes).

\begin{tabular}{|c|c|c|c|c|c|c|}
\hline $\begin{array}{l}\text { Tiempo } \\
\text { posprimera dosis }\end{array}$ & $\begin{array}{l}\text { Número de } \\
\text { nódulos }\end{array}$ & PTH intacta & Calcio & Fósforo & Calcio x Fósforo & $\begin{array}{c}\text { Fosfatasa } \\
\text { alcalina }\end{array}$ \\
\hline Inicial & $3 \pm 0.8944$ & $\begin{array}{c}1130.33 \\
\pm 340.368\end{array}$ & $\begin{array}{c}11.232 \\
\pm 0.56904\end{array}$ & $\begin{array}{c}5.555 \\
\pm 0.645066\end{array}$ & $\begin{array}{c}62.404 \\
\pm 7.6896\end{array}$ & $\begin{array}{c}171 \\
\pm 53.9555\end{array}$ \\
\hline 30 Días & & $\begin{array}{c}543.833 \\
\pm 356.635 \\
\mathrm{P}=0.0077\end{array}$ & $\begin{array}{c}11.258 \\
\pm 0.73112\end{array}$ & $\begin{array}{c}5.948 \\
\pm 0.497732\end{array}$ & $\begin{array}{c}66.908 \\
\pm 6.45756\end{array}$ & No dato \\
\hline 51 Días & & $\begin{array}{c}906.833 \\
\pm 349.27 \\
\mathrm{P}=0.2877\end{array}$ & $\begin{array}{c}10.99 \\
\pm 0.84425\end{array}$ & $\begin{aligned} & 6.082 \\
\pm & 1.01578\end{aligned}$ & $\begin{array}{c}63.312 \\
\pm 11.0939\end{array}$ & $\begin{array}{c}190 \\
\pm 86.0209\end{array}$ \\
\hline 81 Días & & $\begin{array}{c}1006 \\
\pm 368.653 \\
\mathrm{P}=0.5573\end{array}$ & $\begin{aligned} & 11.003 \\
\pm & 0.81835\end{aligned}$ & $\begin{array}{c}5.557 \\
\pm 0.746905\end{array}$ & $\begin{array}{c}60.856 \\
\pm 6.97283\end{array}$ & No dato \\
\hline
\end{tabular}

Tabla 2. Grupo dos. Promedio y desviacion estandar del grupo 2 (tamaño de muestra seis pacientes).

\begin{tabular}{|c|c|c|c|c|c|c|}
\hline $\begin{array}{l}\text { Tiempo } \\
\text { posprimera dosis }\end{array}$ & $\begin{array}{l}\text { Número de } \\
\text { nódulos }\end{array}$ & PTH intacta & Calcio & Fósforo & Calcio x Fósforo & $\begin{array}{c}\text { Fosfatasa } \\
\text { alcalina }\end{array}$ \\
\hline Inicial & $\begin{array}{c}3.167 \\
\pm 0.983192\end{array}$ & $\begin{array}{c}1304.67 \\
\pm 512.031\end{array}$ & $\begin{array}{c}11.068 \\
\pm 0.808466\end{array}$ & $\begin{array}{c}6.225 \\
\pm 0.786251\end{array}$ & $\begin{array}{c}68.611 \\
\pm 7.26241\end{array}$ & $\begin{array}{c}160.167 \\
\pm 44.7188\end{array}$ \\
\hline 51 Días & & $\begin{array}{r}866.833 \\
\pm 286.06\end{array}$ & $\begin{aligned} & 11.068 \\
\pm & 1.04168\end{aligned}$ & $\begin{aligned} & 5.223 \\
\pm & 1.49729\end{aligned}$ & $\begin{aligned} & 58.75 \\
\pm & 18.3854\end{aligned}$ & No dato \\
\hline 75 Días & & $\begin{array}{c}1610.17 \\
\pm 785.748\end{array}$ & $\begin{array}{c}10.692 \\
\pm 1.12824\end{array}$ & $\begin{array}{c}5.587 \\
\pm 0.719073\end{array}$ & $\begin{array}{c}59.939 \\
\pm 11.0134\end{array}$ & $\begin{aligned} & 217.2 \\
\pm & 74.7108\end{aligned}$ \\
\hline 105 Días & & No dato & $\begin{array}{c}10.762 \\
\pm 1.09877\end{array}$ & $\begin{aligned} & 5.927 \\
\pm & 1.13318\end{aligned}$ & $\begin{array}{c}62.747 \\
\pm 14.2529\end{array}$ & No dato \\
\hline 135 Días & & $\begin{array}{c}1280.5 \\
\pm 749.501\end{array}$ & $\begin{array}{c}10.693 \\
\pm 0.750084\end{array}$ & $\begin{aligned} & 5.907 \\
\pm & 1.22078\end{aligned}$ & $\begin{array}{c}61.678 \\
\pm 15.1371\end{array}$ & $\begin{aligned} & 177.5 \\
\pm & 24.7487\end{aligned}$ \\
\hline
\end{tabular}

bas de hipótesis comparando el nivel promedio del PTHi inicial con el PTHi a los 60,120,180 y 240. Se concluyó que con relación a la PTHi inicial hubo reducción significativa en sus valores a los 60 días (EdeT=2.99421, $\mathrm{p}=0.0120941$ ), 120 días $(E d e T=3.15021$, valor de $\mathrm{p}=0.0099)$ y 180 días (EdeT=5.25319, valor de $\mathrm{p}=0.00095)$, pero no a los 240 días $($ EdeT $=0.849681$, valor de $\mathrm{p}=0.214049)$ (Tabla 3$)$.
En las otras variables no se detectaron cambios significativos en el nivel promedio o mediana a través del tiempo.

En ningún grupo se logró reducir los niveles de PTHi a valores considerados de eficacia terapéutica completa (Figura 3). El volumen de las glándulas paratiroides no se modificó significativamente durante el curso del estudio a pesar de las aplicaciones. 
Tabla 3. Grupo 3. Promedio y desviacion estandar del grupo 3 (tamaño de muestra cuatro pacientes).

\begin{tabular}{|c|c|c|c|c|c|c|}
\hline $\begin{array}{l}\text { Tiempo } \\
\text { posprimera dosis }\end{array}$ & $\begin{array}{l}\text { Número de } \\
\text { nódulos }\end{array}$ & PTH intacta & Calcio & Fósforo & Calcio x Fósforo & $\begin{array}{l}\text { Fosfatasa } \\
\text { alcalina }\end{array}$ \\
\hline Inicial & $\begin{array}{l}2,25 \\
\pm 0,5\end{array}$ & $\begin{aligned} & 1478,5 \\
\pm & 266,615\end{aligned}$ & $\begin{array}{c}10,695 \\
\pm 1,06447\end{array}$ & $\begin{array}{c}5,21 \\
\pm 1,39513\end{array}$ & $\begin{array}{r}54,758 \\
\pm 11,007\end{array}$ & $\begin{aligned} & 190,5 \\
\pm & 38,9059\end{aligned}$ \\
\hline 30 Días & & No dato & $\begin{array}{c}11,17 \\
\pm 1,4582\end{array}$ & $\begin{array}{c}4,82 \\
\pm 0,395221\end{array}$ & $\begin{array}{c}53,490 \\
\pm 4,54018\end{array}$ & No dato \\
\hline 60 Días & & $\begin{array}{c}876 \\
\pm 301,458 \\
P=0,0120\end{array}$ & $\begin{array}{c}10,903 \\
\pm 1,91857\end{array}$ & $\begin{array}{c}5,5 \\
\pm 0,588331\end{array}$ & $\begin{array}{c}60,593 \\
\pm 14,9293\end{array}$ & No dato \\
\hline 90 Días & & No dato & $\begin{array}{c}10,748 \\
\pm 1,43778\end{array}$ & $\begin{array}{c}5,415 \\
\pm 0,885983\end{array}$ & $\begin{array}{c}58,956 \\
\pm 15,2823\end{array}$ & $\begin{aligned} & 148,5 \\
\pm & 41,5411\end{aligned}$ \\
\hline 120 Días & & $\begin{array}{c}791 \\
\pm 345,586 \\
\mathrm{P}=0,0099\end{array}$ & $\begin{array}{c}11,063 \\
\pm 1,28102\end{array}$ & $\begin{array}{c}5,305 \\
\pm 0,970103\end{array}$ & $\begin{array}{c}58,108 \\
\pm 8,80159\end{array}$ & No dato \\
\hline 150 Días & & No dato & $\begin{array}{c}10,948 \\
\pm 0,253295\end{array}$ & $\begin{array}{c}\quad 5,605 \\
\pm 1,17236\end{array}$ & $\begin{array}{c}61,247 \\
\pm 12,2231\end{array}$ & No dato \\
\hline 180 Días & & $\begin{array}{c}499,5 \pm 260,463 \\
P=0,00095\end{array}$ & $\begin{array}{c}11 \\
\pm 0,424185\end{array}$ & $\begin{array}{c}5,403 \\
\pm 0,729857\end{array}$ & $\begin{array}{c}59,321 \\
\pm 7,20403\end{array}$ & $\begin{aligned} & 139,25 \\
\pm & 30,5764\end{aligned}$ \\
\hline $\begin{array}{l}210 \text { Días } \\
240 \text { Días }\end{array}$ & & $\begin{array}{c}\text { No dato } \\
\begin{array}{c}1285,75 \\
\pm 367,096 \\
P=0,2140\end{array}\end{array}$ & $\begin{array}{c}11,013 \\
\pm 0,728989 \\
10,235 \\
\pm 0,418688\end{array}$ & $\begin{array}{c}6,025 \\
\pm 2,23001 \\
\quad 4,523 \\
\pm 0,353683\end{array}$ & $\begin{array}{c}65,888 \\
\pm 23,2673 \\
46,299 \\
\pm 4,2511\end{array}$ & $\begin{array}{c}\text { No dato } \\
\begin{array}{c}180,5 \\
\pm 4,9497 \\
\mathrm{~N}=2\end{array}\end{array}$ \\
\hline
\end{tabular}

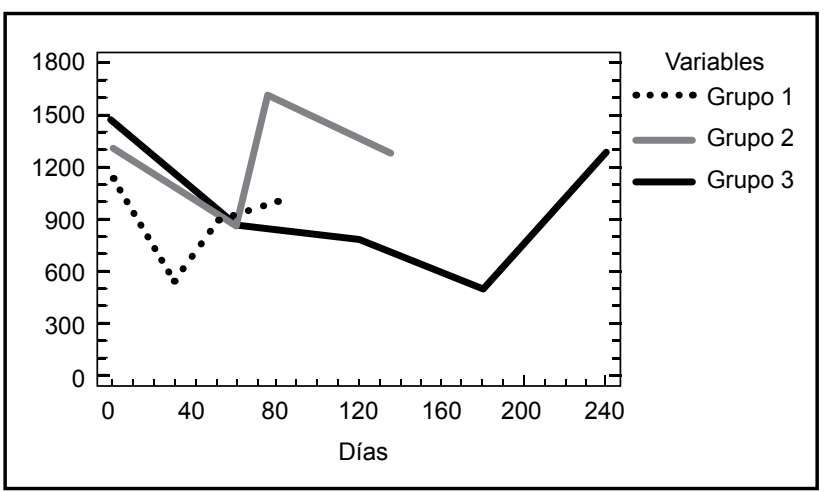

Figura 3. Promedio de PTHi por grupo a través del tiempo. Posprimera dosis.

Efectos colaterales: el procedimiento fue seguro en todos los pacientes, excepto por dolor y sensación de quemazón en el área infiltrada, el cual desapareció en pocos minutos. Disfonía moderada se presentó en dos pacientes, y severa en uno, en el que persistió por tres meses, requiriendo evaluación y terapia por fonoaudiología. En ninguno de los pacientes se presentó hematoma en el área puncionada.

\section{Discusión}

El tratamiento inadecuado de los pacientes con ERC e hiperparatiroidismo secundario lleva inicialmente al crecimiento de las glándulas paratiroideas en forma difusa y policlonal, pero posteriormente avanza a una forma nodular, monoclonal y autónoma, asociándose a disminución del número de receptores de la vitamina $\mathrm{D}$ y calcio cercana al $70 \%(3,4,7)$, lo cual hace que las terapias clásicas para su tratamiento se tornen poco efectivas. Las glándulas paratiroideas con hiperplasia nodular adquieren generalmente un tamaño mayor a $330 \mathrm{~mm}^{3}$, por lo cual pueden ser detectadas por ecógrafos de reciente generación (6). A mayor tamaño glandular se evidencia también mayor posibilidad de refractariedad a terapia con vitamina $\mathrm{D}$, esto ha sido confirmado en diversos estudios, en los cuales se ha observado que cuando el volumen de la glándula paratiroides más grande de las identificadas por ecografía es mayor a $300 \mathrm{~mm}^{3}$ la respuesta al maxacalcitol intravenoso (IV), un agonista selectivo del receptor de la vitamina D es poca $(8,9)$. La asociación de refractariedad e hiperplasia nodular ha sido demostrada por Tominaga y col, quienes estudiaron 187 pacientes con hiperparatiroidismo refractario a maxacalcitol IV, llevados a paratiroidectomía, encontrándose que todos los pacientes refractarios tenían al menos una glándula con hiperplasia nodular, datos correspondientes a volúmenes glandulares mayores de $300 \mathrm{~mm}^{3}$ y pesos glandulares mayores a $500 \mathrm{mg}$ (10). Hallazgos histológicos semejantes se han detectado en pacientes refractarios a calcitriol en pulsos IV, sometidos a paratiroidectomía en el año 1994 (11). En estudios llevados a cabo por Fukagawa y col en pacientes con por lo menos una glándula mayor de $500 \mathrm{~mm}^{3} \mathrm{o} 1 \mathrm{~cm}$ de diámetro se encontró que la gran mayoría habían sido refractarios a terapia con 
calcitriol en pulsos orales $(12,13)$. Katoh también observó que el efecto supresor de una dosis oral de 8 ug de calcitriol en la secreción de PTH fue poco significativo en pacientes con glándulas paratiroides de gran tamaño detectadas por ecografía (14).

El tratamiento de pacientes con hiperplasia nodular es poco exitoso, el etanol intranodular ha dado resultados controvertidos, pero concluimos recientemente que sólo es útil cuando se detecta una sola glándula hiperplásica (5). Teóricamente altos niveles locales de agonistas de la vitamina $\mathrm{D}$ podrían romper la resistencia de las glándulas a su administración sistémica.

La vitamina D activa (calcitriol) intranodular ha resultado efectiva cuando los niveles promedios de PTHi están cercanos a $711 \mathrm{pg} / \mathrm{mL}$, y el volumen glandular a $600 \mathrm{~mm}^{3}$ en siete pacientes tratados por Kitaoka y cols (15); resultados semejantes obtiene este grupo en reporte del año 2003 en 20 pacientes sometidos a tres dosis en una semana (16). Shiizaki y col también obtienen buenos resultados al aplicar diariamente calcitriol intranodular por cinco a diez días consecutivos (17), al igual que Nakanishi y col en nueve pacientes a los que se les administró una sola dosis de 200-300\% del volumen calculado de la glándula paratiroides (18).

Agonistas selectivos del receptor de la vitamina D podrían ejercer un mayor efecto local y con menos efectos sistémicos (hipercalcemia, hiperfosfatemia); al respecto Shiizaki y cols utilizaron el 22-oxacalcitriol (22-oxa-1.25 dihidroxivitamina D3 (maxacalcitol)) por inyección intranodular semanal por seis dosis en 20 pacientes con hiperplasia nodular y lograron una significativa reducción en los valores de PTHi luego de sólo 12 semanas de seguimiento (19). Un esquema de administración semanal por cuatro a seis semanas logra también controlar el hiperparatiroidismo y además reducir el tamaño de las glándulas paratiroides (20). El riesgo de lesión del nervio laríngeo es mínimo con la vitamina D comparado con lo publicado para el etanol.

Pero los resultados de la terapia con agonistas de receptor de la vitamina $\mathrm{D}$ intraglandular en pacientes con hiperparatiroidismo refractario no siempre son satisfactorios. En Brasil Barros Gueiros JE y col reportan nueve pacientes con ERC en terapia hemodialítica e hiperparatiroidismo secundario con PTH intacta mayor a 450 y una o más glándulas paratiroides con volumen mayor a $500 \mathrm{~mm}^{3}$, a quienes se les aplicó calcitriol intraglandular tres veces por semana por dos semanas para un total de seis inyecciones por glándula (en caso de más de una glándula se inyectaron todas al mismo tiempo); la cantidad inyectada fue acorde con $80 \%$ del volumen calculado de la glándula por ecografía, observándose incapacidad en todos los pacientes para lograr valores satisfactorios de PTH entre 200 - 300 pg/mL; 81.2\% de los pacientes estudiados por histopatología posterior a la paratiroidectomía presentaron hiperplasia nodular (21).

Los resultados variables de la aplicación de vitamina $\mathrm{D}$ intranodular pueden ser explicados porque a la fecha no está claramente establecido cuándo está indicada, cual es la forma ideal de aplicarla, ni el mejor intervalo.

En Colombia no contamos con calcitriol de uso parenteral, ni con maxacalcitol, pero sí con otro agonista selectivo del receptor de la vitamina D, el paricalcitol (Zemplar®) en ampollas para uso intravenoso al finalizar la hemodiálisis, y que ha demostrado en diversos estudios su capacidad de disminuir la secreción de paratohormona en pacientes con hiperparatiroidismo, sin inducir severa hipercalcemia ni hiperfosfatemia (22). No hay a la fecha publicaciones que demuestren su utilidad intranodular en pacientes con hiperparatiroidismo refractario.

En nuestro estudio encontramos que la administración intranodular de paricalcitol logra reducir significativamente los niveles de PTHi (aunque no a valores considerados de eficacia terapéutica completa), siempre y cuando se administre en grupos de dos glándulas con un intervalo de tiempo no superior a una semana, o por administración mensual simultánea de todas las glándulas a un intervalo de cada mes. El medicamento es muy bien tolerado, y su efecto a largo plazo en un esquema de aplicación mensual debería ser evaluado en un futuro.

Concluimos que en pacientes con hiperparatiroidismo secundario refractario, la ecografía de cuello es una herramienta importantísima para tomar una conducta terapéutica. Pacientes con un sólo adenoma pueden beneficiarse de agonistas selectivos del receptor de la vitamina $\mathrm{D}$ oral o parenteral (según su tipo de terapia dialítica) asociado o no a cinacalcet. Si no presentan buena respuesta terapéutica y no tienen contraindicación quirúrgica proceder con paratiroidectomía, en caso contrario considera etanol intranodular. Para pacientes en los cuales se identifica más de un adenoma paratiroideo se debe también intentar la misma terapia medicamentosa propuesta anteriormente, aunque la posibilidad de repuesta es menor al $30 \%$, principalmente si alguno tiene un volumen mayor a $500 \mathrm{~mm}^{3}$; la paratiroidectomía quirúrgica sería la siguiente elección. Para pacientes que no sean candidatos a terapia quirúrgica, la aplicación seriada cada mes de paricalcitol intranodular en cada uno de los nódulos puede constituir una muy buena alternativa terapéutica.

\section{Ninguno \\ Fuentes de apoyo y conflicto de interés}

\section{Referencias}

1. Block GA, Klassen PS, Lazarus JM, Ofsthun N, Lowrie EG, Chertow GM Mineral metabolism, mortality and morbidity in maintenance hemodialysis patients. J Am Soc Nephrol 2004; 15: 2208-2218.

2. Hirai T, Nakashima A, Takasugi N, Yorioka N. Association of nodular hyperplasia with resistance to Cinacalcet therapy for secondary hyperparathyroidism in hemodialysis patients. TherApher Dial 2010; 14: 577-582.

3. Fukuda N, Tanaka H, Tominaga Y, Fukagawa M, Kurokawa K, Seiko Y Decreased 1,25-dihydroxyvitamin D3 receptor density associated with a more severe form of parathyroid hyperplasia in chronic uremic patients. J Clin Invest 1993; 92:1436-1443

4. Gogusev J, Duchambon P, Hory B, Giovannini M, Goureau Y, Sarfati E, et al Depressed expression of calcium receptor in parathyroid gland tissue of patients with hyperparathyroidism. Kidney Int 1997; 51: 328-336. 
5. Restrepo CA, Castillo CE,Sanz MF, Velez C. La eficacia del etanol intraglandular en hiperparatiroidismo secundario se limita a una sola glándula hiperplásica. Acta Med Colomb 2013; 38: 61-67.

6. Restrepo C A, Santacruz D, Castillo C E, Chacon J A. Detección de hiperplasia de paratiroides por ultrasonografía y correlación con signos clínicos y de laboratorio en pacientes con enfermedad renal crónica. Rev Colomb Radiol 2011; 22: 3341-3347.

7. Tominaga Y, Kohara S, Namii Y, Nagasaka T, Haba T, Uchida K, et al. Clonal analysis of nodular parathyroid hyperplasia in renal hyperparathyroidism. World J Surg 1993; 20: 744-750.

8. Okuno S, Ishimura E, Kitatani K Et al. Relationship between parathyroid gland size and responsiveness to maxacalcitol therapy in patients with secondary hyperparathyroidism. Nephrol Dial Transpl 2003; 18: 2613-2621.

9. Tominaga Y, Inaguma D, Matsuoka S, et al. PTG studygroup. Is the volume of the parathyroid gland a predictor of Maxacalcitol response in advanced secondary hyperparathyroidism? Ther Apher Dial 2006; 10: 198-204.

10. Tominaga Y, Matsuoka S, Sato T, et al. Clinical features and hyper plastic patterns of parathyroidglands in hemodialysis patients with advanced secondary hyperparathyroidism refractory to maxacalcitol treatment and required parathyroidectomy. Ther Apher Dial 2007; 11: 266-273.

11. Tominaga Y, Numano M, Uchida K, et al. Parathyroidectomy for patients with renal hyperparathyroid is refractory to calcitriol pulse therapy. J Bone Miner Met 1994; 12 (suppl 1): S99-S104.

12. Fukagawa M, Kazama JJ, Shigematsu T. Management of patients with advanced secondary hyperparathyroidism: the Japanese approach. Nephrol Dial Transplant 2002; 17: 1553-1557.

13. Fukagawa M, Kitaoka M, Yi $\mathbf{H}$ et al. Serial evaluation of parathyroid size by ultrasonography is an ther use fulmarker for the long-term prognosis of calcitriol pulse therapy in chronic dialysis patients. Nephron 1994; 68: 221-228.
14. Katoh N, Nakayama M, Shigematsu $T$ et al. Presence of sonographically detectable parathyroidglands can predict resistance to oral pulsed-dose calcitriol treatment of secondary hyperparathyroidism. Am J Kidney Dis 2000; 35: 465-468.

15. Kitaoka, Fukagawa M, Fukuda N, Ogata E. Direct injection of calcitriol into enlarged parathyroid glands in chronic dialysis patients with severe parathyroid hyperfunction. Nephrology 1995; 1: 563-567.

16. Kitaoka M, Onoda N, Kitamura H, Koiwa F, Tanaka M, Fukagawa M. Percutaneous calcitriol injection therapy (PCIT) for secondary hyperparathyroidism: multicentre trial. Nephrol Dial Transplant 2003; 18 (suppl 3): 38-41.

17. Shiizaki K, Negi S, Mizobuchi M, et al. Effect of percutaneous calcitriol injection therapy on secondary hyperparathyroidism in uraemic patients. Nephrol Dial Transplant 2003; 18 (suppl 3): 42-46.

18. Nakanishi S, Yano S, Nomura R, et al. Efficacy of direct injection of calcitriol into the parathyroid glands in uraemic patients with moderate to severe secondary hyperparathyroidism. Nephrol Dial Transplant 2003; 18 (suppl 3): 47-49.

19.Shiizaki K, Hatamura I, Negi S, et al. Percutaneous maxacalcitol injection therapy regresses hyperplasia of parathyroid and induces apoptosis in uremia. Kidney Int 2003; 64: 992-1003.

20. Saito A, Matsumoto Y, Oyama Y, Asaka M, Yokoyama H. Effectiveness ok weekly percutaneous maxacalcitol injection therapy in patients with secondary hyperparathyroidism. Ther Apher Dial 2010; 14: 98-103.

21. de Barros Gueiros JE, Chammas MC, Gerhard R, et al. Percutaneous ethanol (PEIT) and calcitrol (PCIT) injection therapy are ineffective in treatings ever secondary hyperparathyroidism. Nephrol Dial Transplant. 2004; 19: 657-663.

22. Biggar P, Kovarik, Klauser-Braun R, et al. Paricalcitol treatment of secondary hyperparathyroidism in hemodialys is patients: A German-Austrain, single-arm, open-label, prospective, noninterventional, observational study. Nephron Clin Pract 2014; 17: 39-50. 